\title{
Dosimetric characterization of medical linear accelerator Photon and Electron beams for the treatment accuracy of cancer patients
}

Motiur Rahman 1, M Shamsuzzaman 2, ${ }^{*}$, Manoshi Sarker 4, Abdul Jobber 1, Mohsin Mia 1, Asish Kumar Bairagi 1, Musfika Ahmed ${ }^{1}$, Shohel Reza ${ }^{1}$, Sadiq R Malik ${ }^{1}$, MMH Bhuiyan ${ }^{2}$, ASM Habibullah Khan ${ }^{3}$ and MKA Khan ${ }^{4}$

${ }^{1}$ Delta Hospital Limited, Mirpur-1, Dhaka-1216.

2 Institute of Nuclear Science and Technology, Atomic Energy Research Establishment Bangladesh Atomic Energy Commission, Dhaka-1349.

3 Planning and Development Division Bangladesh Atomic Energy Commission, Dhaka-1207.

${ }^{4}$ Department of Physics, Jagannath University, Dhaka-1000.

World Journal of Advanced Engineering Technology and Sciences, 2021, 03(01), 041-059

Publication history: Received on 04 May 2021; revised on 11 August 2021; accepted on 13 August 2021

Article DOI: https://doi.org/10.30574/wjaets.2021.3.1.0046

\begin{abstract}
In radiotherapy treatment planning process, quality assessment (QA) is indispensable for achieving accuracy and avoidance of treatment errors. In this perspective, present study focused on the Photon and Electron beams characterization of a medical linear accelerator (LINAC) to ascertain dosimetric QA in Absolute and Reference dosimetry. In this connection, the beam outputs were investigated in terms of $D_{\max }$ and $D_{w, \max }$ (dose at depth $d_{\max }$ ) in absolute dosimetry for Photon and Electron beams, respectively. In accordance with the measured $D_{\max }$ and $D_{w}$, max parameters, Photon and Electron beam outputs were standardized to ensure standard output of $1 \mathrm{cGy} / \mathrm{MU}$. In reference dosimetry, the parametric evaluation was performed for dosimetric QA in terms of percent depth dose (PDD), beam profile flatness and symmetry, output factors: $S_{c p}, S_{c}$, $S_{\mathrm{p}}$ with varying field size (FS) ranging from $4 \times 4 \mathrm{~cm}^{2}$ to $40 \times 40 \mathrm{~cm}^{2}$ normalized at FS $10 \times 10 \mathrm{~cm}^{2}$ for the $6 \mathrm{MV}$ and $10 \mathrm{MV}$ Photon beams. The measured PDDs at $10 \mathrm{~cm}$ depth $\left(D_{10}\right)$ were found to be $66.8 \%$ and $73.6 \%$ for $6 \mathrm{MV}$ and $10 \mathrm{MV}$ Photon beams, respectively, with significantly small deviation of $1 \%$ and $0.8 \%$ in comparison with an international PDD protocol of British Journal of Radiology-25 (BJR -25). In the case of Electron beams characterization, PDD was verified with $10 \times 10 \mathrm{~cm}^{2}$ cone/applicator, beam profile flatness and symmetry were analyzed at the field sizes ranging from $6 \times 6 \mathrm{~cm}^{2}$ to $25 \times 25 \mathrm{~cm}^{2}$ normalized at $10 \times 10 \mathrm{~cm}^{2}$ cone/applicator, and Electron cone ratios were investigated for a given cone/applicator relative to the $15 \times 15 \mathrm{~cm}^{2}$ one for the 6, 9, 12, $15 \mathrm{MeV}$ Electron energies. The PDDs of all the Electron beams revealed reasonable consistency with manufacturer's estimations of $90 \%, 80 \%$, and 50\% PDDs at various depths of ionization.
\end{abstract}

Keywords: Percent Depth Dose; Water Phantom; Ionization Chamber; Photon; Electron; Field Size

\section{Introduction}

Cancer is the second leading cause of death in the world after cardiovascular diseases which claims the lives of millions across the world [1]. Nevertheless, nowadays millions of cancer patients extend their life due to early identification and treatment. Generally, cancer is treated by surgery, radiotherapy or chemotherapy either separately or in combination of any of the above treatment modalities. Recently immune-therapy, photodynamic-therapy, monoclonal antibodytherapy or other methods are used as an alternative or complementary technique in the treatment of cancer. The choice of therapy depends upon the location and grade of the tumor and the stage of the disease, as well as the general state of

\footnotetext{
${ }^{*}$ Corresponding author: M Shamsuzzaman

Institute of Nuclear Science and Technology, Atomic Energy Research Establishment Bangladesh Atomic Energy Commission, Dhaka1349.
}

Copyright (C) 2021 Author(s) retain the copyright of this article. This article is published under the terms of the Creative Commons Attribution Liscense 4.0. 
the patient (performance status). However, among various cancer therapy modalities, more than $65 \%$ of patients are primarily treated with radiotherapy. The aim of radiotherapy is to cure or locally control the cancer diseases while minimizing complications in normal tissues using ionizing radiation e.g., X-rays, Gamma ray Photon beams, and Electron beams, etc. The ultimate goal of radiotherapy is to cure the patient without any damage to other organs. In radiotherapy, every cancer patient deserves to receive the best possible accuracy in prescribed dose for the malignant tumor to achieve a desired precession in curative treatment or palliation in long-term tumor control. Currently, radiotherapy performs a key role as one of the useful treatment modalities to ensure the best outcome in cancer treatment procedure.

Nowadays, external beam radiotherapy with ionizing radiation has become one of several modalities used for cancer treatment, along with chemotherapy and surgery. It is estimated as a vital part of cancer management, since around $50 \%$ of patients receiving radiotherapy in their treatment [2]. In the middle of the twentieth century, megavoltage energies became available through the use of Betatrons, Cobalt- 60 gamma rays, and LINACs. Among these modalities, concurrently developed medical LINACs became the most widely used radiation source in modern radiotherapy. Almost all developed countries are using LINAC as a basic treatment machine. In a LINAC, energy is higher and charged particles are accelerated in a straight line, so it can treat deep seated tumor with better doses. In using LINAC, dose rate remains same because when the machine is on work only then radiation generates while no decay in shutdown. The conceivable minimum field size in LINAC is $0.5 \times 0.5 \mathrm{~cm}^{2}$, hence, every small area can be treated by using LINAC although the accurate dosimetry of small fields in sub-centimeter range used in modern treatment techniques makes the measurement difficult due to steep dose gradient, lack of charge particle equilibrium, detector size as well as the partial occlusion of the radiation beam $[3,4,5]$. Nevertheless, a LINAC customizes high energy X-rays to conform to a tumor's shape with a view to deliver a large, uniform X-ray fields in conventional therapy to destroy cancer cells while sparing surrounding normal tissue [6]. The advancement of LINAC technology has improved the precision and efficiency of radiation therapy. Conventional LINACs are relatively safe in the sense that once machine is off there is no radiation in the room. Thus, after delivering the prescribed dose there will be no X-rays produced [7].

The purpose of applying LINACs in radiotherapy is to cure or improve the quality of life of a cancer patient through the accurate delivery of a radiation dose to a prescribed target volume [8]. However, there will always be some uncertainty in the dose to be delivered. This uncertainty in dose is due to errors and physical limitations that may occur at different steps in the radiotherapy treatment process. Since patient care is always the first priority in radiotherapy, hence corresponding quality assurance $(\mathrm{QA})$ is a prerequisite that has been introduced for long been in place.

Among all treatment modalities, as the specialized LINAC machines are being used for achieving higher accuracy in radiotherapy, hence, must be properly maintained and checked to ensure quality treatment. Radiation is an invisible energy source and therefore errors are not simply detected during delivery. Therefore, regular QA of the machine through measurements and checks are a vital part of radiotherapy. The necessity of QA is mainly to ensure that radiation dose is delivered correctly. In this connection, machine's beam characteristics needs to be measured and compared to tolerance levels (unacceptable in the long term) and action levels (unacceptable at any time). Presently, in Bangladesh, most of the government and private hospitals are using LINACs for external beam radiotherapy with high energy photon and Electron beams. In such perspective, quality assessment (QA) of LINAC is prerequisite to ensure the accuracy of the prescribed dose from the Photon and Electron beams [9]. Hence, the QA of a LINAC machine needs to be performed prior to patient treatment in order to maintain the treatment accuracy as per standard protocols $[9,10]$. The main purpose of QA is to ensure the consistency of quality of a machine, patient safety, deliverance of right dose to the patients, the mechanical or software errors. To fulfill this QA requirements, the process of commissioning a LINAC for clinical use includes comprehensive measurements of dosimetric parameters that are necessary to validate the treatment planning systems (TPS), to be used to select optimal radiation modality and treatment technique for individual patients [11]. Therefore, it is essential to have a minimum data set which includes Percentage Depth Dose (PDD), Dosimetric Profile, and Output characterization for a series of field sizes.

Keeping this in mind, some basic dosimetric parameters like Beam outputs, PDDs, Profile flatness and symmetry, and Output factors were measured with different FS for the Photon and Electron beams of LINAC (D-2300CD) machine. The prime objective of this study is to verify the dosimetric beam profile for the evaluation of Photon and Electron beam characteristics to ensure the treatment accuracy. In line with this objective, the measured PDD profiles were authenticated in terms of the standards of the BJR-25 [12]. The main aim of this study is to measure dosimetric parameters in order to minimize the uncertainties of the treatment process so as to avoid unnecessary exposure to critical organs. 


\section{Material and methods}

\subsection{Experimental Instrumentation}

A medical LINAC machine (D-2300CD) was undertaken for radiation beam quality assessment. This type of medical LINAC is commonly used to treat malignant lesions anywhere in or on the patient's body. Thus, the radiation beam quality assessment is a prerequisite to maintain the desired treatment accuracy. In this perspective, the dosimetry profile of this medical LINAC was investigated in this study. This LINAC has dual energy mode of Photon and Electron beam facility. It has two Photon beams of 6 and $10 \mathrm{MV}$ beam potential, and four Electron beams of 6, 9, 12 and 15 MeV energies. The advantage of applying the 6 and 10 megavoltage X-ray Photon beams is that they can easily penetrate the body to reach deep-seated tumors, including those of the prostate, colon, liver or ovary. On the other hand, the abovementioned megavoltage Electron beams are less penetrating than X-rays but create a more-uniform field of radiation. These Electron beams do not irradiate the tissues which lies below the tumor; they are used for skin cancers and tumors close to the surface, as well as for head and neck tumors, where it is vital to avoid the brain or spinal cord under the cancer. A photographic view of the analyzed LINAC (D-2300CD) is shown in Fig. 1 (a), and the schematic diagram of a typical medical LINAC is shown in Fig. 1 (b).
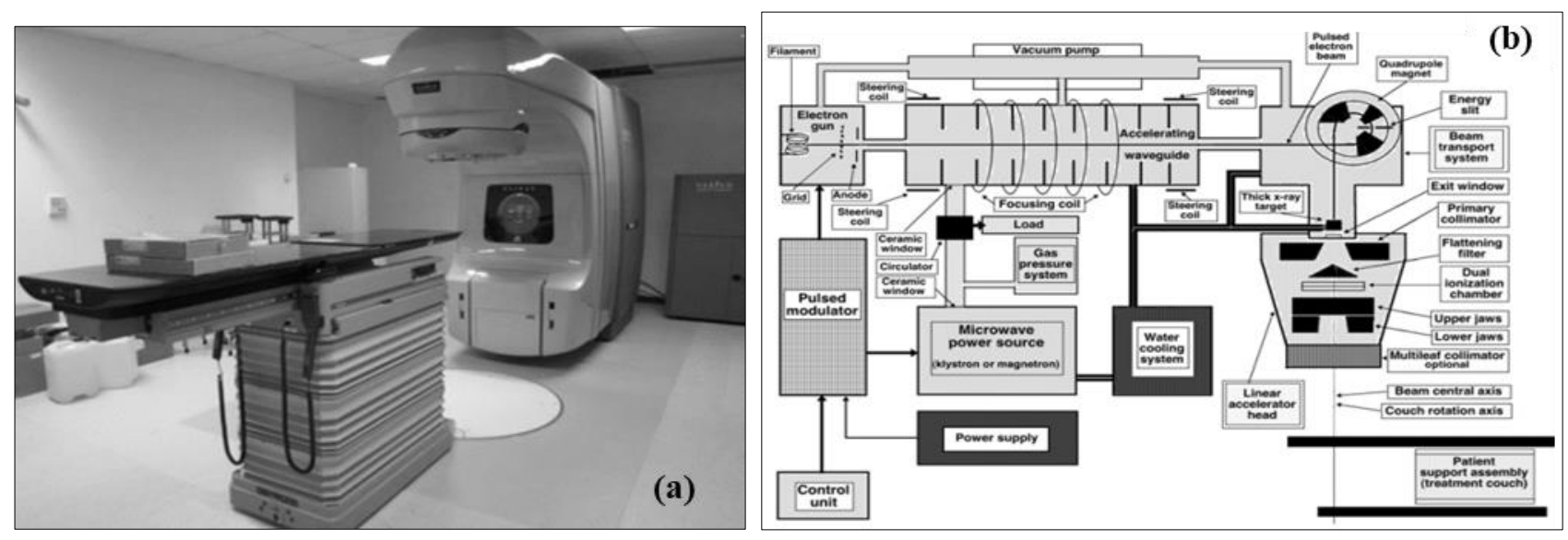

Figure 1 (a) Photographic view of the analyzed LINAC (D-2300CD) (b) Schematic diagram of a typical medical LINAC machine

The major modules of this LINAC (D-2300CD) are the Drive stand, Gantry, Modulator cabinet, Treatment couch and Control console. The multileaf collimator (MLC) of this LINAC is the tertiary collimator system which is positioned just below the level of the standard upper and lower adjustable jaws. There are 80 leaf MLC in the collimator of this LINAC and these collimators are static. For tertiary systems, the collimator jaws are used in concert with the leaves to shape the X-ray fields. The X-jaws move along the leaf direction. On the other hand, Y-jaws are strategically placed at the upper and lower borders of the field. To facilitate the Electron beam therapy, the auxiliary system of the presented LINAC consists of several basic systems that are not directly involved with Electron acceleration, rather these systems make the acceleration possible for clinical operation with Electron beam. The Electron beam transport system brings the pulsed high-energy Electron beam from the accelerating waveguide onto the target in the X-ray therapy mode and onto the scattering foil in the Electron therapy mode [13].

\subsection{Experimental Technique}

The dosimetry feature of the analyzed medical LINAC (D-2300 CD) was investigated in the present study to authenticate the QA profile. The dosimetric verification of the radiation beam is a prerequisite prior to commission the clinical trial. This is because, in commissioning of external beam radiotherapy with a LINAC, it needs to take into account of all radiation beam characteristics. The beam output statistics is required to be organized into a dosimetry data book, to be entered into a computerized treatment planning system (TPS) for dosimetric quality assurance (QA). Consequently, based on this dosimetric QA parameters, treatment plan and treatment procedures are verified. In order to acquire the radiation beam characteristics, the basic dosimetric parameters, namely, PDDs, profile flatness and symmetry, output factors, and transmission factors were experimentally measured. These basic dosimetric parameters were measured for both Photon beams ( 6 and $10 \mathrm{MV}$ ) and Electron beams (6, 9, 12 and 15MeV) with different field size(s). Experimental measurements were performed in a Three-Dimensional (3D) computer-controlled water phantom (IBA RFA300) with using the waterproof cylindrical type Farmer ionization chamber (0.65cc, IBA FC 65 P\#1787) and an IBA Compact 
Chamber (0.13cc IBA CC13) coupled with an IBA electrometer (DOSE1\#13527). This electrometer (DOSE1\#13527) provides significant precision in data acquisition as it exceeds the recommendations of the IEC 60731 [14]. Finally, these dosimetric equipment were deployed under the exposure arrangement of the LINAC machine to verify its Photon and Electron beams characteristics. The main intention of measuring these dosimetric parameters is to minimize the uncertainties in the treatment process with a view to avoid unnecessary exposure to the adjacent critical organs. The analysis of the dosimetric parameters were performed by using IBA Planning software. To verify the accuracy, measured PDD profiles were authenticated in terms of the BJR-25 protocol. The purpose of this process is to substantiate the measured dosimetric characteristics of a LINAC machine in terms of standard protocols.

\subsection{Dose Assessment Instrumentation}

A large size motorized 3D water phantom (IBA RFA300) was used for the Absolute and Reference dosimetry of the Photon and Electron radiation beams with vertical beam incidence. This system is based on the large-size water tank with a scanning range of $480 \times 408 \times 500 \mathrm{~mm}$. This 3D phantom has the detector moving range up to $480 \mathrm{~mm}$ with precise 3D stainless steel moving mechanism driven by three calibration-free, high-speed stepper motors. This device includes three stepper motors for a detector positioning speed of $50 \mathrm{~mm} / \mathrm{s}$ and a positioning accuracy of $\pm 0.1 \mathrm{~mm}$. It consists of stable $20 \mathrm{~mm}$ thick acrylic walls and etched lines for precise tank alignment. In dose measurement with this phantom, a cylindrical type IBA farmer ionization chamber (0.65cc, IBA FC 65 P\#1787) was mounted to IBA RFA300 phantom for the absolute dosimetry and reference dosimetry as well as measuring the tray transmission factor and MLC transmission factor. In the measurement process of total output factor $\left(S_{c p}\right)$, collimator scatter factor $\left(S_{c}\right)$, and phantom scatter factor $\left(S_{p}\right)$, an IBA Compact Chamber (0.13cc IBA CC13) was used as a standard chamber for clinical applications with high reproducibility in a water phantom. In the experimental dosimetric data acquisition process, a DOSE 1 (S/N 13527) electrometer was coupled with the aforesaid waterproof chambers. This electrometer is high-precision reference class device for the measurements of absorbed dose which significantly exceeds the recommendations of the IEC 60731 [14]. The present dosimetry process was performed by complying with the dosimetry protocol of American Association of Physicists in Medicine (AAMP): AAPM TG-51[15].

\section{Results and discussion}

The dosimetric parameters of the LINAC beam characteristics were verified to ascertain consistency with clinical applications. Measurements were performed through systematic and sequential approach. Essential procedures were followed methodically to ensure the accuracy in dose delivery as a prerequisite of a LINAC machine to be certified and commissioned for the treatment of cancer patients. This condition essentially requires to be fulfilled to satisfy the clinical data accuracy as per standard protocols' tolerance values of the beam parameters. In this connection, the breakthrough of this study demonstrated the assurance of precise dose delivery in clinical application. Therefore, the dosimetric QA breakthrough of the present LINAC beams reasonably complied with the tolerance limits of the standard dosimetrty protocols $[9,10]$ as an imperative to ensure treatment quality with accurate dose delivery of a LINAC machine in any cancer center. The detailed dosimetric QA approach is discussed in the subsequent section:

\subsection{Photon Beam Characterization}

\subsubsection{Maximum Depth Dose Measurement}

The beam output was investigated in terms of $D_{\max }$ to characterize the Photon beams. In this process, depth doses $\left(D_{w}\right)$ at $10 \mathrm{~cm}$ depth $\left(D_{10}\right)$ were measured, and in accordance with this $D_{10}$, maximum depth doses $\left(D_{\max }\right)$ were estimated for the $6 \mathrm{MV}$ and $10 \mathrm{MV}$ Photon beams with reference field size $10 \times 10 \mathrm{~cm}^{2}$ and source-to-surface distance (SSD) $100 \mathrm{~cm}$ as per AAPM TG-51 protocol [15]. The purpose of this measurement is to standardize the Photon beam outputs. The procedural sequences of this measurement at respective Photon beams are described in the subsequent section:

\section{MV Photon Beam}

The depth dose $\left(D_{w}\right)$ of $6 \mathrm{MV}$ Photon beam measured at $10 \mathrm{~cm}$ depth of water was found to be $0.683352 \mathrm{cGy} / \mathrm{MU}$. In accordance with this, the measured dose maximum $\left(D_{\max }\right)$ was found to be $1.02298 \mathrm{cGy} / \mathrm{MU}$. One of the main goals of this study is to ascertain the standard dose of $1 \mathrm{cGy} / \mathrm{MU}$, hence to ensure this, the observed data of the meter reading $\mathrm{M}_{10}$ was tuned at $1.3455 \times 10^{-8} \mathrm{C}$ from $1.4401 \times 10^{-8} \mathrm{C}$; consequently, $D_{\max }$ was set to the dose of $1.0002 \mathrm{cGy} / 1 \mathrm{MU}$ which is significantly equal to the required standard dose of $1 \mathrm{cGy} / \mathrm{MU}$. 
10 MV Photon Beam

The depth dose $\left(D_{w}\right)$ of $10 \mathrm{MV}$ Photon beam measured at $10 \mathrm{~cm}$ depth of water was found to be $0.74722 \mathrm{cGy} / \mathrm{MU}$. The measured dose maximum $\left(D_{\max }\right)$ was found to be $1.0124 \mathrm{cGy} / \mathrm{MU}$. Like 6MV beam, in order to ascertain a standard dose of $1 \mathrm{cGy} / \mathrm{MU}$, the observed data of the meter reading $\mathrm{M}_{10}$ was tuned at $1.4800 \times 10^{-8} \mathrm{C}$ from $1.5961 \times 10^{-8} \mathrm{C}$; consequently, $D_{\max }$ was set to the dose of $0.9988 \mathrm{cGy} / \mathrm{MU}$ which indicates a good agreement with the required standard dose of 1 cGy/MU.

\subsubsection{Verification of Percentage Depth Dose (PDD) for 6MV and 10 MV beams}

The PDD scan was performed with field sizes (FS) ranging from $4 \times 4 \mathrm{~cm}^{2}$ to $40 \times 40 \mathrm{~cm}^{2}$ using the 3D IBA RFA300 phantom. The demonstrated PDDs were measured for the FS of $10 \times 10 \mathrm{~cm}^{2}$ and SSD of $100 \mathrm{~cm}$. These PDDs were acquired from the scanning data at different water depths in the 3D phantom with the mounted ionization chamber (0.65cc, IBA FC 65 P\#1787) for FS of $10 \times 10 \mathrm{~cm}^{2}$. The PDD profiles scanned at 6 and $10 \mathrm{MV}$ Photon beams are shown in Fig. 2 (a, b).

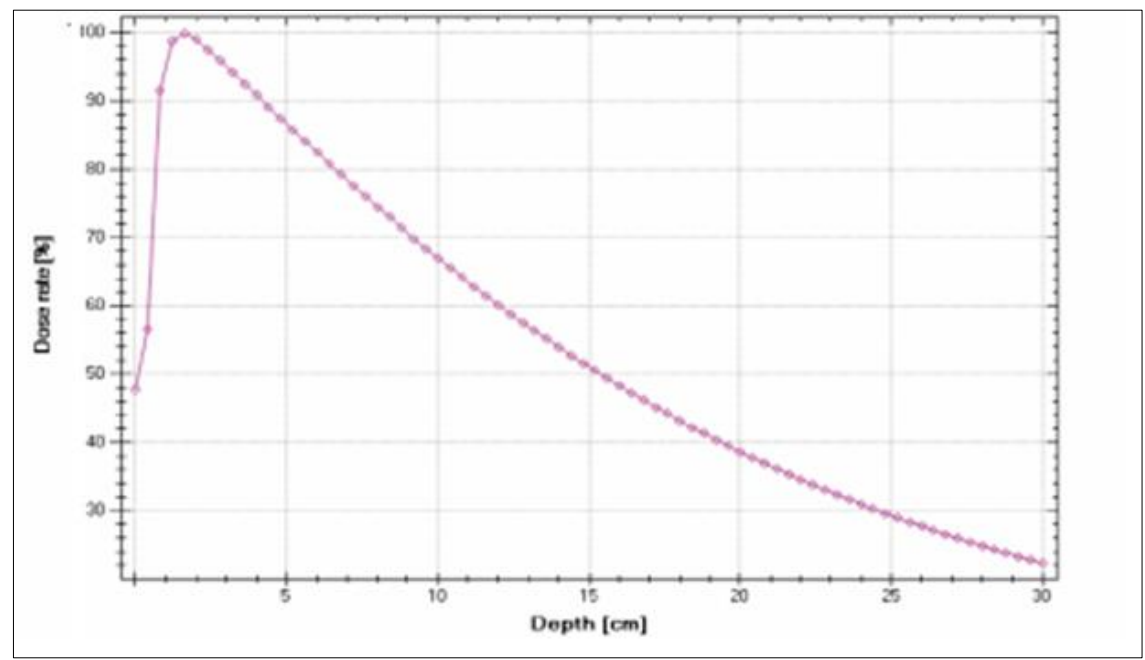

(a) PDD profile scanned at $6 \mathrm{MV}$

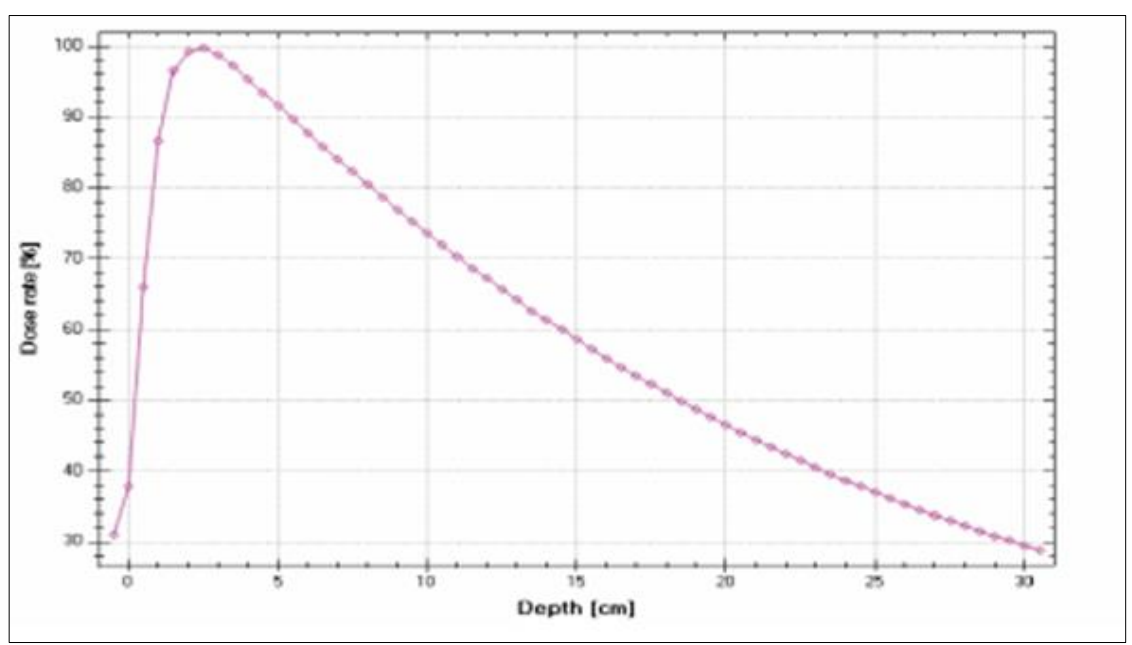

(b) PDD profile scanned at $10 \mathrm{MV}$

Figure 2 The PDD profiles scanned at (a) $6 \mathrm{MV}$ and (b) $10 \mathrm{MV}$

The measured PDDs at $10 \mathrm{~cm}$ depth $\left(D_{10}\right)$ were found to be $66.8 \%$ and $73.61 \%$ for $6 \mathrm{MV}$ and $10 \mathrm{MV}$ Photon beams, respectively that indicates a small deviation of $1 \%$ and $0.8 \%$ in comparison with an international standard PDD protocol of BJR-25 [12]. 
The corresponding depth vs measured PDDs for 6 MV and $10 \mathrm{MV}$ are presented in Table 1 and Table 2 respectively.

Table 1 The depth vs measured PDDs for 6 MV

\begin{tabular}{|c|c|c|c|}
\hline Depth(cm) & Measured PDD & Clinical \%DD (From BJR-25) & $\mathbf{\%} \boldsymbol{\Delta}$ \\
\hline 1.5 & 99.9 & 100 & 0.1 \\
\hline 5 & 86.6 & 86.9 & 0.3 \\
\hline 10 & 66.8 & 67.5 & 1.0 \\
\hline 15 & 50.6 & 51.7 & 1.9 \\
\hline 20 & 38.4 & 39.3 & 2.3 \\
\hline
\end{tabular}

Table 2 The depth vs measured PDDs for 10MV

\begin{tabular}{|c|c|c|c|}
\hline Depth(cm) & Measured PDD & Clinical PDD (From BJR-25) & $\mathbf{\%} \boldsymbol{\Delta}$ \\
\hline 2.5 & 99.9 & 100 & 0.1 \\
\hline 5 & 91.9 & 91.4 & 0.5 \\
\hline 10 & 73.6 & 73.0 & 0.8 \\
\hline 15 & 58.3 & 57.8 & 0.85 \\
\hline 20 & 46.2 & 45.6 & 1.3 \\
\hline
\end{tabular}

\subsubsection{Profile Flatness and Symmetry of Photon beams}

Flatness and Symmetry are main parameters in determining the quality of the Photon and Electron beams produced by linear accelerators which can be defined in accordance with IEC60976 [16]. To maintain the consistent beam profile, the flatness and symmetry accuracy needs to be within the recommended tolerance limits of $\pm 2 \%$ and $\pm 3 \%$ respectively [9]. Thus, the flatness and symmetry values in the central $80 \%$ field width of the measured profile, as defined during machine commissioning, should not deviate from the baseline by more than the tolerance values [9]. In the current study, the Photon beams were scanned at 6MV and 10MV to obtain the lateral profile which offers a scope to check the flatness and symmetry of the beam. The flatness and symmetry profile of the scanned Photon beams are evident in Fig. $3(a, b)$. The observed variation in the verified flatness and symmetry profile of the scanned beams are presented in Table 3. The flatness was determined according to the following Equation 1.

$$
\text { Flatness }(\%)=\frac{D_{\max }-D_{\min }}{D_{\max }+D_{\min }} \times 100 \% \text { (1) }
$$

where, $D_{\max }$ and $D_{\min }$ are the maximum and minimum dose along the profile within the core $80 \%$ of the field size.

Similarly, the symmetry was determined according to the following Equation 2.

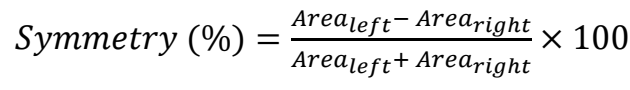

where, $A_{\text {left }}$ and $A_{\text {right }}$ are the areas bounded by the profile on the left and right of the beam central axis.

The Profile flatness and symmetry were evaluated with FS $10 \times 10 \mathrm{~cm}^{2}$ for $6 \mathrm{MV}$ and $10 \mathrm{MV}$ Photon beams. The Profile flatness with FS $10 \times 10 \mathrm{~cm}^{2}$ for $6 \mathrm{MV}$ and $10 \mathrm{MV}$ Photon beams were found to be $1.1 \%$ and $1.1 \%$. The symmetry with FS $10 \times 10 \mathrm{~cm}^{2}$ for $6 \mathrm{MV}$ and $10 \mathrm{MV}$ Photon energies were found to be $1 \%$ and $0.7 \%$ as presented in Table 3 . In accordance with the AAPM TG-40, the flatness and symmetry tolerances for $6 \mathrm{MV}$ and $10 \mathrm{MV}$ Photon are up to $\pm 2 \%$ and $\pm 3 \%$, respectively. Therefore, the flatness and symmetry of Profiles for 6MV and 10MV Photon beams with reference FS $\left(10 \times 10 \mathrm{~cm}^{2}\right)$ are found within the recommended range of the AAPM TG-40 scale. 


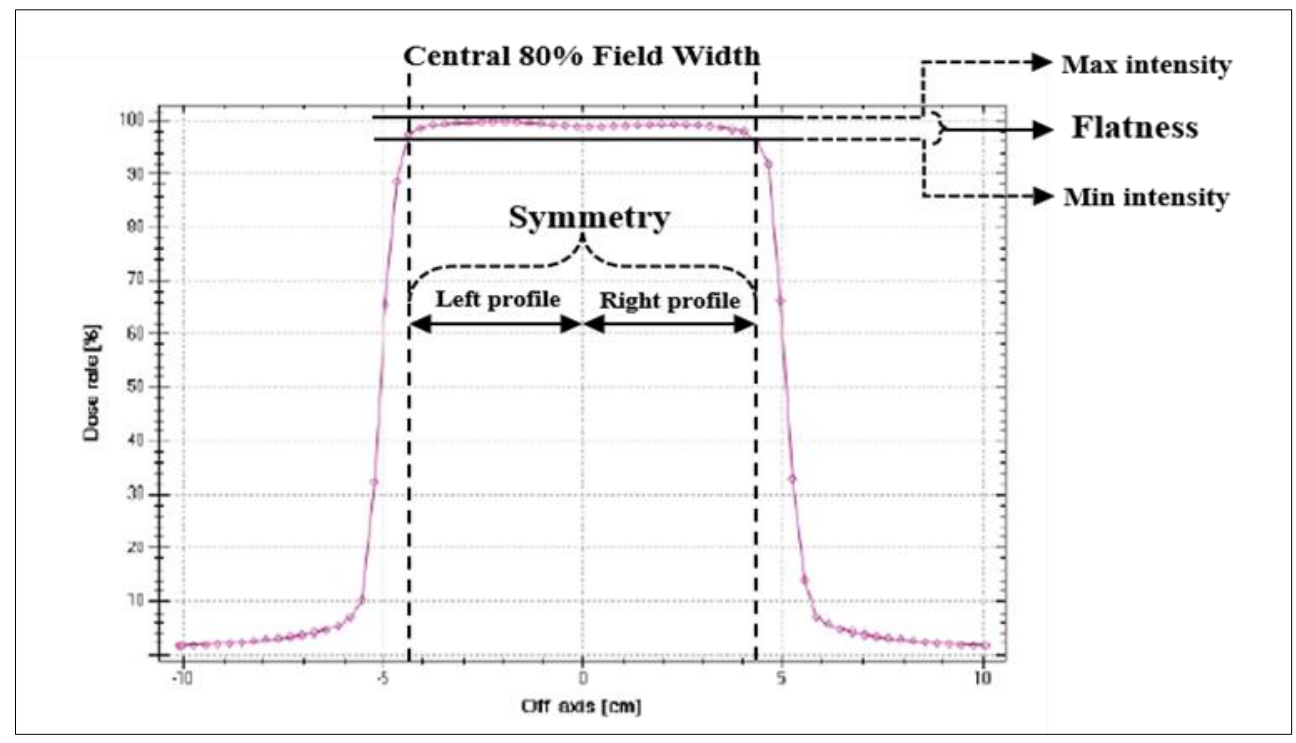

(a) Beam profile scanned at 6MV

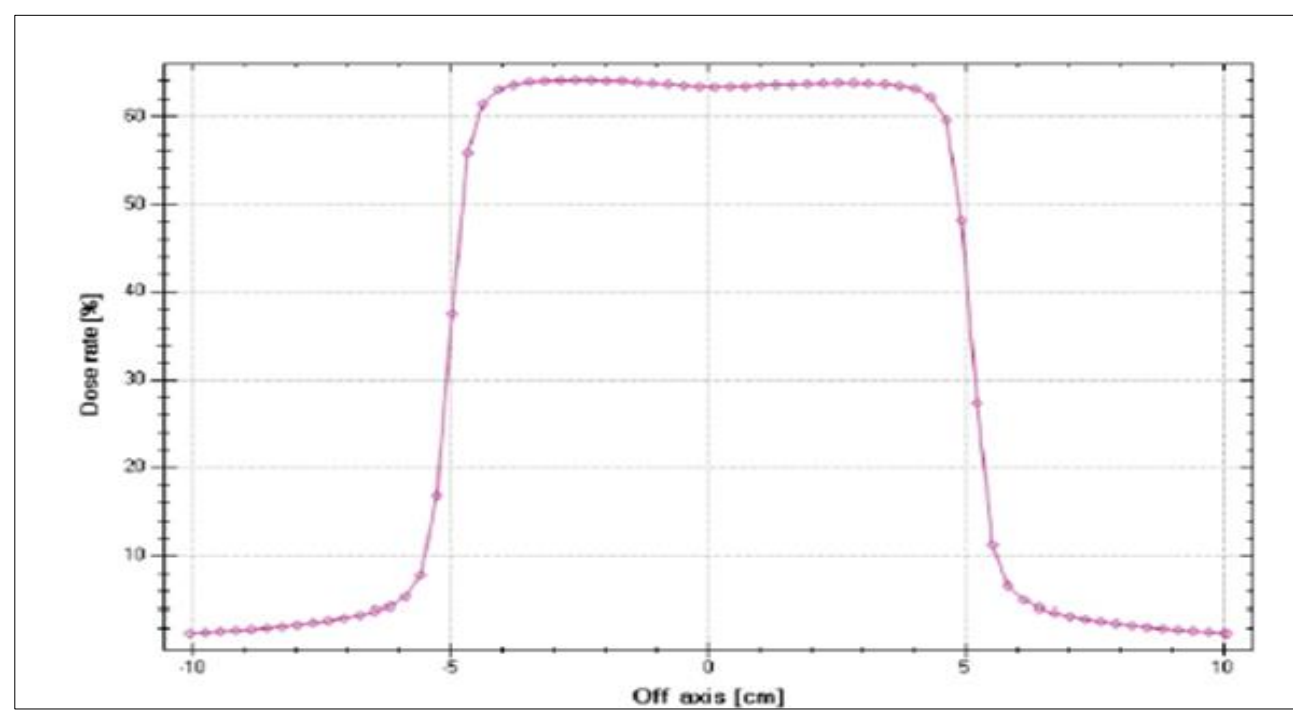

(b) Beam profile scanned at $10 \mathrm{MV}$

Figure 3 Beam profile of Flatness and Symmetry of Photon beams (a) scanned at 6MV and (b) scanned at10MV Correspondingly, evaluated flatness and symmetry for the Photon beams of 6MV and $10 \mathrm{MV}$ are presented in Table 3.

Table 3 The Flatness and Symmetry of the Photon beams

\begin{tabular}{|c|c|c|c|c|}
\hline Beam voltage (MV) & Field Size & Symmetry & Flatness & Penumbra \\
\hline 6 & $10 \times 10$ & $1 \%$ & $1.1 \%$ & $0.65 \mathrm{~cm}-0.66 \mathrm{~cm}$ \\
\hline 10 & $10 \times 10$ & $0.7 \%$ & $1.1 \%$ & $0.66 \mathrm{~cm}-0.64 \mathrm{~cm}$ \\
\hline
\end{tabular}

\subsubsection{Verification of various output factors}

Various output factors, namely total output factor $\left(S_{c p}\right)$, collimator scatter factor $\left(S_{c}\right)$ and phantom scatter factor $\left(S_{p}\right)$ were verified with different FS (s) ranging from at $4 \times 4 \mathrm{~cm}^{2}$ to $40 \times 40 \mathrm{~cm}^{2}$ normalized at $10 \times 10 \mathrm{~cm}^{2} \mathrm{FS}$ for the $6 \mathrm{MV}$ and 10 MV Photon beams. 
Total output factor $\left(S_{c p}\right)$

The total output factor $\left(S_{c p}\right)$ is defined as a ratio of the absorbed dose at the reference depth $\mathrm{d}$ for any field size $\mathrm{A} \times \mathrm{A} \mathrm{cm}^{2}$ to the dose at the same depth $d$ and SSD for the reference field $\left(10 \times 10 \mathrm{~cm}^{2}\right)$. The total output factors were measured at reference depth $d_{\max }$ in a large 3D water phantom at $100 \mathrm{~cm}$ SAD (i.e., $98.5 \mathrm{~cm}$ SSD) for $6 \mathrm{MV}$ and $100 \mathrm{~cm}$ SAD (i.e., 97.6 cm SSD) for $10 \mathrm{MV}$ using an IBA Compact Chamber (0.13cc IBA CC13) and an IBA electrometer (D0SE1\#13527). The measured data are presented in Table 4 and Table 5 for 6 MV and 10 MV Photon beams, respectively.

Table 4 Measured total output factors $\left(S_{c p}\right)$ for $6 \mathrm{MV}$ at $100 \mathrm{MU}$ and dose rate: $300 \mathrm{MU} / \mathrm{min}$

\begin{tabular}{|c|c|c|c|c|}
\hline Field Size & $\mathbf{M}_{\mathbf{1} \times \mathbf{1}^{\mathbf{0}-\mathbf{8}} \mathbf{C}}$ & $\mathbf{M}_{\mathbf{2} \times \mathbf{1 0}} \mathbf{- 8} \mathbf{C}$ & Average & $\boldsymbol{S}_{\boldsymbol{c} \boldsymbol{p}}$ \\
\hline $4 \times 4$ & 2.0050 & 2.0060 & 2.0055 & 0.9236 \\
\hline $6 \times 6$ & 2.0770 & 2.0760 & 2.0765 & 0.9563 \\
\hline $8 \times 8$ & 2.1300 & 2.1310 & 2.1305 & 0.9811 \\
\hline $10 \times 10$ & 2.1720 & 2.1710 & 2.1715 & 1.0000 \\
\hline $12 \times 12$ & 2.2060 & 2.2060 & 2.2060 & 1.0159 \\
\hline $15 \times 15$ & 2.2450 & 2.2450 & 2.2450 & 1.0338 \\
\hline $20 \times 20$ & 2.2920 & 2.2920 & 2.2920 & 1.0555 \\
\hline $25 \times 25$ & 2.3260 & 2.3270 & 2.3265 & 1.0714 \\
\hline $30 \times 30$ & 2.3590 & 2.3590 & 2.3590 & 1.0863 \\
\hline $35 \times 35$ & 2.3840 & 2.3840 & 2.3840 & 1.0979 \\
\hline $40 \times 40$ & 2.4010 & 2.4010 & 2.4010 & 1.1057 \\
\hline
\end{tabular}

Table 5 Measured total output factors (Scp) for $10 \mathrm{MV}$ at $100 \mathrm{MU}$ and dose rate: 300MU/min

\begin{tabular}{|c|c|c|c|c|}
\hline Field Size & $\mathbf{M}_{\mathbf{1} \times \mathbf{1 0}^{-\mathbf{8}} \mathbf{C}}$ & $\mathbf{M}_{\mathbf{2} \times \mathbf{1 0}^{-\mathbf{8}} \mathbf{C}}$ & Average & $\boldsymbol{S}_{\boldsymbol{c}}$ \\
\hline $4 \times 4$ & 2.0260 & 2.0250 & 2.0255 & 0.9112 \\
\hline $6 \times 6$ & 2.1160 & 2.1160 & 2.1160 & 0.9519 \\
\hline $8 \times 8$ & 2.1770 & 2.1780 & 2.1775 & 0.9795 \\
\hline $10 \times 10$ & 2.2230 & 2.2230 & 2.2230 & 1.0000 \\
\hline $12 \times 12$ & 2.2620 & 2.2620 & 2.2620 & 1.0175 \\
\hline $15 \times 15$ & 2.3000 & 2.3020 & 2.3010 & 1.0351 \\
\hline $20 \times 20$ & 2.3470 & 2.3480 & 2.3475 & 1.0560 \\
\hline $25 \times 25$ & 2.3810 & 2.3810 & 2.3810 & 1.0711 \\
\hline $30 \times 30$ & 2.4140 & 2.4140 & 2.4140 & 1.0859 \\
\hline $35 \times 35$ & 2.4390 & 2.4390 & 2.4390 & 1.0972 \\
\hline $40 \times 40$ & 2.4590 & 2.4580 & 2.4585 & 1.1059 \\
\hline
\end{tabular}

Collimator scatter factor $\left(\mathrm{S}_{\mathrm{c}}\right)$

The collimator scatter factor (Sc) is commonly called the output factor and can be defined as the ratio of the output in air for a given field to that for a reference field $(10 \times 10 \mathrm{~cm} 2)$. This Sc was measured using an IBA Compact Chamber (0.13cc IBA CC13) with a buildup cap of size large enough to provide maximum dose buildup for the given energy 
beam. The Sc was investigated by measuring the relative ionization in air for both $6 \mathrm{MV}$ and 10 MV Photon beams as presented in Table 6 and Table 7, respectively.

Table 6 Measured collimator scatter factors (Sc) for 6MV at SSD=100 and 100MU

\begin{tabular}{|c|c|c|c|c|}
\hline Field Size $\left(\mathbf{c m}^{\mathbf{}} \mathbf{)}\right.$ & $\mathbf{M}_{\mathbf{1} \times \mathbf{1 0}} \mathbf{- 8} \mathbf{C}$ & $\mathbf{M}_{\mathbf{2} \times \mathbf{1 0}^{-\mathbf{8}} \mathbf{C}}$ & Average & $\boldsymbol{S}_{\boldsymbol{c p}}$ \\
\hline $4 \times 4$ & 0.3820 & 0.3820 & 0.3820 & 0.9550 \\
\hline $6 \times 6$ & 0.3900 & 0.3900 & 0.3900 & 0.9750 \\
\hline $8 \times 8$ & 0.3960 & 0.3960 & 0.3960 & 0.9900 \\
\hline $10 \times 10$ & 0.4000 & 0.4000 & 0.4000 & 1.0000 \\
\hline $12 \times 12$ & 0.4040 & 0.4040 & 0.4040 & 1.0100 \\
\hline $15 \times 15$ & 0.4070 & 0.4070 & 0.4070 & 1.0175 \\
\hline $20 \times 20$ & 0.4110 & 0.4110 & 0.4110 & 1.0275 \\
\hline $25 \times 25$ & 0.4130 & 0.4130 & 0.4130 & 1.0325 \\
\hline $30 \times 30$ & 0.4150 & 0.4150 & 0.4150 & 1.0375 \\
\hline $35 \times 35$ & 0.4170 & 0.4170 & 0.4170 & 1.0425 \\
\hline $40 \times 40$ & 0.4190 & 0.4190 & 0.4190 & 1.0475 \\
\hline
\end{tabular}

Table 7 Measured collimator scatter factors (Sc) for $10 \mathrm{MV}$ at SSD=100 and 100MU

\begin{tabular}{|c|c|c|c|c|}
\hline Field Size $\left(\mathbf{c m}^{\mathbf{}} \mathbf{)}\right.$ & $\mathbf{M}_{\mathbf{1} \times \mathbf{1 0}^{-\mathbf{8}} \mathbf{C}}$ & $\mathbf{M}_{\mathbf{2}} \times \mathbf{1 0}^{-\mathbf{8}} \mathbf{C}$ & Average & $\boldsymbol{S}_{\boldsymbol{c}}$ \\
\hline $4 \times 4$ & 0.4500 & 0.4570 & 0.4505 & 0.9514 \\
\hline $6 \times 6$ & 0.4590 & 0.4610 & 0.4600 & 0.9715 \\
\hline $8 \times 8$ & 0.4680 & 0.4690 & 0.4685 & 0.9894 \\
\hline $10 \times 10$ & 0.4730 & 0.4740 & 0.4735 & 1.0000 \\
\hline $12 \times 12$ & 0.4780 & 0.4780 & 0.4780 & 1.0095 \\
\hline $15 \times 15$ & 0.4820 & 0.4820 & 0.4820 & 1.0180 \\
\hline $20 \times 20$ & 0.4860 & 0.4860 & 0.4860 & 1.0264 \\
\hline $25 \times 25$ & 0.4900 & 0.4900 & 0.4900 & 1.0348 \\
\hline $30 \times 30$ & 0.4920 & 0.4920 & 0.4920 & 1.0391 \\
\hline $35 \times 35$ & 0.4960 & 0.4960 & 0.4960 & 1.0475 \\
\hline
\end{tabular}

Phantom Scatter Factor $\left(\mathrm{S}_{\mathrm{p}}\right)$

The phantom scatter describes the influence of the scatter originating in the phantom only, which can be quantified by the phantom scatter factor $\left(S_{p}\right)$. The $S_{p}$ is defined as the phantom scatter dose contribution for a specified collimator defined field size and a specified field size at the phantom surface, normalized to unity for the reference irradiation setup. It is derived from the total scatter correction factor $\left(S_{c p}\right)$, divided by the collimator scatter correction factor $\left(S_{c}\right)$ for the same collimator defined field size. Thus, $S_{p}$ is the ratio of total output factor $\left(S_{c p}\right)$ to collimator scatter factor $\left(S_{c}\right)$, denoted as $S_{p}=\frac{S_{c p}}{S_{p}}$. The measured phantom scatter factors $\left(S_{p}\right)$ for $6 \mathrm{MV}$ and $10 \mathrm{MV}$ Photons beams are presented in Table 8 and Table 9 respectively. 
Table 8 Measured phantom scatter factors (Sp) for 6MV at SSD = $100 \mathrm{~cm}$ and $100 \mathrm{MU}$

\begin{tabular}{|c|c|c|c|}
\hline Field Size $\left(\mathbf{c m}^{2}\right)$ & $\boldsymbol{S}_{\boldsymbol{c}}$ & $\boldsymbol{S}_{\boldsymbol{c}}$ & $\boldsymbol{S}_{\boldsymbol{p}}=\boldsymbol{S}_{\boldsymbol{C P}} / \boldsymbol{S}_{\boldsymbol{C}}$ \\
\hline $4 \times 4$ & 0.9236 & 0.9550 & 0.9671 \\
\hline $6 \times 6$ & 0.9563 & 0.9750 & 0.9808 \\
\hline $8 \times 8$ & 0.9811 & 0.9900 & 0.9910 \\
\hline $10 \times 10$ & 1.0000 & 1.0000 & 1.0000 \\
\hline $12 \times 12$ & 1.0159 & 1.0100 & 1.0058 \\
\hline $15 \times 15$ & 1.0338 & 1.0175 & 1.0161 \\
\hline $20 \times 20$ & 1.0555 & 1.0275 & 1.0272 \\
\hline $25 \times 25$ & 1.0714 & 1.0325 & 1.0377 \\
\hline $30 \times 30$ & 1.0863 & 1.0375 & 1.0471 \\
\hline $35 \times 35$ & 1.0979 & 1.0425 & 1.0531 \\
\hline $40 \times 40$ & 1.1057 & 1.0475 & 1.0555 \\
\hline
\end{tabular}

Table 9 Measured phantom scatter factors (Sp) for $10 \mathrm{MV}$ at SSD $=100 \mathrm{~cm}$ and $100 \mathrm{MU}$

\begin{tabular}{|c|c|c|c|}
\hline Field Size $\left(\mathbf{c m}^{2}\right)$ & $\boldsymbol{S}_{\boldsymbol{c} p}$ & $\boldsymbol{S}_{\boldsymbol{c}}$ & $\boldsymbol{S}_{\boldsymbol{p}}=\boldsymbol{S}_{\boldsymbol{C P}} / \boldsymbol{S}_{\boldsymbol{c}}$ \\
\hline $4 \times 4$ & 0.9112 & 0.9514 & 0.9577 \\
\hline $6 \times 6$ & 0.9519 & 0.9715 & 0.9798 \\
\hline $8 \times 8$ & 0.9795 & 0.9894 & 0.9900 \\
\hline $10 \times 10$ & 1.0000 & 1.0000 & 1.0000 \\
\hline $12 \times 12$ & 1.0175 & 1.0095 & 1.0080 \\
\hline $15 \times 15$ & 1.0351 & 1.0180 & 1.0168 \\
\hline $20 \times 20$ & 1.0560 & 1.0264 & 1.0288 \\
\hline $25 \times 25$ & 1.0711 & 1.0348 & 1.0350 \\
\hline $30 \times 30$ & 1.0859 & 1.0391 & 1.0451 \\
\hline $35 \times 35$ & 1.0972 & 1.0475 & 1.0474 \\
\hline $40 \times 40$ & 1.1059 & 1.0475 & 1.0558 \\
\hline
\end{tabular}

\subsubsection{Tray Transmission Factor}

The tray transmission factor (TTF) is defined as a ratio of the dose in a phantom with and without a tray in the beam for the same number of monitor units delivered at a fixed SSD and depth in phantom. In this study, tray transmission factor was measured at $10 \mathrm{~cm}$ depth in water for $100 \mathrm{~cm}$ SSD and $10 \times 10 \mathrm{~cm}^{2} \mathrm{FS}$ using IBA farmer type ionization chamber (0.65cc IBA FC 65 P\#1787) and IBA electrometer Dose 1(S/N: 13527). The measured transmission factor was found within $\pm 2 \%$ of the manufacturer's tolerance value. The verified tray transmission factors for $6 \mathrm{MV}$ and $10 \mathrm{MV}$ Photon beams are presented in Table 10. 
Table 10 Verified tray transmission factors (TF) for 6 MV and 10 MV Photon beams

\begin{tabular}{|c|c|c|c|c|c|}
\hline \multirow{2}{*}{$\begin{array}{l}\text { Beam } \\
\text { voltage } \\
\text { (MV) }\end{array}$} & \multirow{2}{*}{$\begin{array}{l}\text { Output: } \\
\text { without tray }\end{array}$} & \multicolumn{2}{|l|}{ Output: with Tray } & \multirow{2}{*}{ Average } & \multirow{2}{*}{ TTF } \\
\cline { 3 - 6 } & $\mathbf{M}_{\mathbf{1} \times \mathbf{1 0}^{-8} \mathbf{C}}$ & $\mathbf{M}_{\mathbf{2} \times \mathbf{1 0}^{-8} \mathbf{C}}$ & & \\
\hline 6 & 1.408 & 1.364 & 1.364 & 1.364 & 0.97 \\
\hline 10 & 1.563 & 1.526 & 1.526 & 1.526 & 0.98 \\
\hline
\end{tabular}

\subsubsection{Multileaf Collimator Transmission Factor}

Multileaf collimator (MLC) transmission factor (TF) was measured at $10 \mathrm{~cm}$ depth in water with $100 \mathrm{~cm} \mathrm{SSD} \mathrm{and} 10 \times 10$ $\mathrm{cm}^{2} \mathrm{FS}$ coupling an IBA Farmer type ionization chamber (0.65cc IBA FC65P\#1787) and IBA electrometer Dose1 (S/N: 13527). The MLC transmission factor was found to be within $\pm 2 \%$ of the manufacturer's tolerance value. The verified MLC transmission factors for 6MV and 10 MV Photon beams are presented in Table 11.

Table 11 Verified Multileaf Collimator (MLC) Transmission Factor (TF)

\begin{tabular}{|c|c|c|c|c|c|}
\hline \multirow{2}{*}{$\begin{array}{c}\text { Beam } \\
\text { voltage } \\
\text { (MV) }\end{array}$} & Output & \multicolumn{2}{|c|}{ Output with MLC } & \multirow{2}{*}{ Average } & MLCTF \\
\cline { 3 - 4 } & MLC & $\mathrm{M}_{1} \times 10^{-8} \mathrm{C}$ & $\mathrm{M}_{2} \times 10^{-8} \mathrm{C}$ & & \\
\hline 6 & 1.3925 & 0.019 & 0.019 & 0.019 & 0.0136 \\
\hline 10 & 1.5325 & 0.025 & 0.024 & 0.0245 & 0.0160 \\
\hline
\end{tabular}

\subsubsection{Wedge Transmission Factor}

Wedge transmission factor (WTF) was measured at $10 \mathrm{~cm}$ depth in water with $100 \mathrm{~cm} \mathrm{SSD}$ and $10 \times 10 \mathrm{~cm}^{2} \mathrm{FS}$ for the $6 \mathrm{MV}$ and $10 \mathrm{MV}$ beam quality. In both cases, a Farmer type IBA chamber and was used in the measurement process. The monitor unit (MU) was set at $100 \mathrm{MU}$ in both beam qualities, and the open field readings were recorded as $1.4016 \times 10^{-}$ ${ }^{8} \mathrm{C}$ and $1.556 \times 10^{-8} \mathrm{C}$ for $6 \mathrm{MV}$ and $10 \mathrm{MV}$ Photon beams respectively. The measured WTFs for $\mathrm{M} \mathrm{V}$ and $10 \mathrm{M} \mathrm{V}$ are 6 presented in Table 12 and Table 13, respectively.

Table 12 Measured wedge transmission factors for 6MV

\begin{tabular}{|c|c|c|c|c|c|c|}
\hline $\begin{array}{l}\text { Angle } \\
\text { (degree) }\end{array}$ & $\begin{array}{l}\text { Wedge } \\
\text { Position }\end{array}$ & $\mathrm{M}_{1 \times 10^{-8}} \mathrm{C}$ & $\mathrm{M}_{2} \times 10^{-8} \mathrm{C}$ & Average & $\begin{array}{l}\text { Average } \\
\text { (In \& Out) }\end{array}$ & WTF \\
\hline 15 & In & 1.072 & 1.072 & 1.072 & \multirow{4}{*}{1.074} & \multirow{4}{*}{0.767} \\
\hline 15 & out & 1.077 & 1.077 & 1.077 & & \\
\hline 15 & Left & 1.074 & 1.074 & 1.074 & & \\
\hline 15 & Right & 1.075 & 1.075 & 1.075 & & \\
\hline 30 & In & 0.863 & 0.863 & 0.863 & \multirow{2}{*}{0.867} & \multirow{2}{*}{0.619} \\
\hline 30 & Out & 0.871 & 0.871 & 0.871 & & \\
\hline 45 & In & 0.684 & 0.684 & 0.684 & \multirow{2}{*}{0.689} & \multirow{2}{*}{0.492} \\
\hline 45 & Out & 0.694 & 0.694 & 0.694 & & \\
\hline 60 & In & 0.562 & 0.563 & 0.563 & \multirow{2}{*}{0.571} & \multirow{2}{*}{0.407} \\
\hline 60 & Out & 0.578 & 0.579 & 0.579 & & \\
\hline
\end{tabular}


Table 13 Measured wedge transmission factors for 10MV

\begin{tabular}{|c|c|c|c|c|c|c|}
\hline $\begin{array}{c}\text { Angle } \\
\text { (degree) }\end{array}$ & $\begin{array}{l}\text { Wedge } \\
\text { Position }\end{array}$ & $\mathrm{M}_{1 \times 10^{-8}} \mathrm{C}$ & $\mathrm{M}_{2 \times 10^{-8} \mathrm{C}}$ & Average & $\begin{array}{c}\text { Average } \\
\text { (In \&Out) }\end{array}$ & WTF \\
\hline 15 & In & 1.247 & 1.246 & 1.2465 & \multirow{2}{*}{1.249} & \multirow{2}{*}{0.803} \\
\hline 15 & Out & 1.252 & 1.252 & 1.252 & & \\
\hline 30 & In & 1.039 & 1.039 & 1.039 & \multirow{2}{*}{1.043} & \multirow{2}{*}{0.670} \\
\hline 30 & Out & 1.046 & 1.046 & 1.046 & & \\
\hline 45 & In & 0.812 & 0.813 & 0.813 & \multirow{2}{*}{0.818} & \multirow{2}{*}{0.526} \\
\hline 45 & Out & 0.823 & 0.823 & 0.823 & & \\
\hline 60 & In & 0.675 & 0.675 & 0.675 & \multirow{2}{*}{0.683} & \multirow{2}{*}{0.439} \\
\hline 60 & Out & 0.691 & 0.691 & 0.691 & & \\
\hline
\end{tabular}

\subsection{Electron Beam Characterization}

\subsubsection{Depth Dose Measurement}

The Electron beam output was investigated in terms of maximum depth dose $\left(D_{w, \max }\right)$. In this regard, depth doses $\left(D_{w}\right.$, $\left.d_{r e f}\right)$ were measured at reference depth $d_{\text {ref }}$ for the all the Electron energies of $6,9,12,15 \mathrm{MeV}$ with $10 \times 10 \mathrm{~cm}^{2} \mathrm{cone}$ and $100 \mathrm{~cm}$ SSD according to TG-51 protocol [15]. Then, $D_{w, \max }$ was determined in accordance with the measured $D_{w, d r e f}$ The purpose of this measurement is to standardize the Electron beam outputs. The stepwise process of this measurement technique for respective Electron energies are described in the subsequent section:

\section{$6 \mathrm{MeV}$ Electron Beam}

The depth dose per monitor unit $\left(D_{w}\right.$, dref $\left./ \mathrm{MU}\right)$ of $6 \mathrm{MeV}$ Electron beam measured at $d_{\text {ref }}$ in water was found to be 0.9792 cGy/MU. In accordance with this, the measured Dose/MU at $d_{\max }\left(D_{w, \max }\right)$ was found to be $0.9008 \mathrm{cGy} / \mathrm{MU}$. The main focus of this study is to ensure a standard dose $\left(D_{w, \max }\right)$ of $1 \mathrm{cGy} / \mathrm{MU}$ as beam output, hence to ensure this, the observed data of the meter reading $M_{d r e f}$ was tuned to $2.241 \times 10^{-8} \mathrm{C}$ from $2.189 \times 10^{-8} \mathrm{C}$, consequently $D_{w, \max }$ was set to the dose of $1.0031 \mathrm{cGy} / \mathrm{MU}$, which is perfectly meet the required standard dose of $1 \mathrm{cGy} / \mathrm{MU}$.

\section{$9 \mathrm{MeV}$ Electron Beam}

The depth dose per monitor unit $\left(D_{w}\right.$, dref $\left./ \mathrm{MU}\right)$ of $9 \mathrm{MeV}$ Electron beam measured at $d_{\text {ref }}$ in water was found to be 1.0268 cGy/MU. In accordance with this, the measured Dose/MU at $d_{\max }\left(D_{w, \max }\right)$ was found to be 1.0339 cGy/MU. To achieve the standard dose $\left(D_{w, \max }\right)$ of $1 \mathrm{cGy} / \mathrm{MU}$ as beam output, the observed data of the meter reading $M_{d r e f}$ was tuned to $2.2096 \times 10^{-8} \mathrm{C}$ from $2.2733 \times 10^{-8} \mathrm{C}$, consequently $D_{w, \max }$ was set to the dose of $1.0049 / \mathrm{MU}$ cGy, which indicates a good agreement with the required standard dose of $1 \mathrm{cGy} / \mathrm{MU}$.

\section{$12 \mathrm{MeV}$ Electron Beam}

The depth dose per monitor unit $\left(D_{w, d r e f} / \mathrm{MU}\right)$ of $12 \mathrm{MeV}$ Electron beam measured at $d_{\text {ref }}$ in water was found to be 1.0595 cGy/MU. In accordance with this, the measured Dose/MU at $d_{\max }\left(D_{w, \max }\right)$ was found to be $1.0599 \mathrm{cGy} / \mathrm{MU}$. To achieve the standard dose $\left(D_{w, \max }\right)$ of $1 \mathrm{cGy} / \mathrm{MU}$ as beam output, the observed data of the meter reading $M_{d r e f}$ was tuned to $2.207 \times 10^{-8} \mathrm{C}$ from $2.336 \times 10^{-8} \mathrm{C}$, consequently $D_{w \text {, max }}$ was set to the dose of $1.0014 \mathrm{cGy} / \mathrm{MU}$, which perfectly satisfies the required standard dose of $1 \mathrm{cGy} / \mathrm{MU}$.

\section{$15 \mathrm{MeV}$ Electron Beam}

The depth dose per monitor unit $\left(D_{w, d r e f} / \mathrm{MU}\right)$ of $15 \mathrm{MeV}$ Electron beam measured at $d_{\text {ref }}$ in water was found to be 1.0588 cGy/MU. In accordance with this, the measured Dose/MU at $d_{\max }\left(D_{w, \max }\right)$ was found to be $1.0604 \mathrm{cGy} / \mathrm{MU}$. To achieve the standard dose $\left(D_{w, \max }\right)$ of $1 \mathrm{cGy} / \mathrm{MU}$ as beam output, the observed data of the meter reading $M_{d r e f}$ was tuned to $2.2193 \times 10^{-8} \mathrm{C}$ from $2.357 \times 10^{-8} \mathrm{C}$, consequently $D_{w, \max }$ was set to the dose of $0.9985 \mathrm{cGy} / \mathrm{MU}$, which ensures a good agreement with the required standard dose of $1 \mathrm{cGy} / \mathrm{MU}$. 


\subsubsection{Percent Depth Dose (PDD)}

The percent depth doses were scanned with $10 \times 10 \mathrm{~cm}^{2}$ cones for all Electron energies with the Scanditronix scanning system. In this process an IBA Compact Chamber (0.13cc IBA CC13) was used for PDD scanning process as per AAMP TG-25 protocol to produce depth dose curves [17]. The experimental observations were consistent with the manufacturer's specifications. The PDD curves of Electron beams scanned at 6, 9, 12, $15 \mathrm{MeV}$ are shown in Fig. 4 (a, b, c, d).

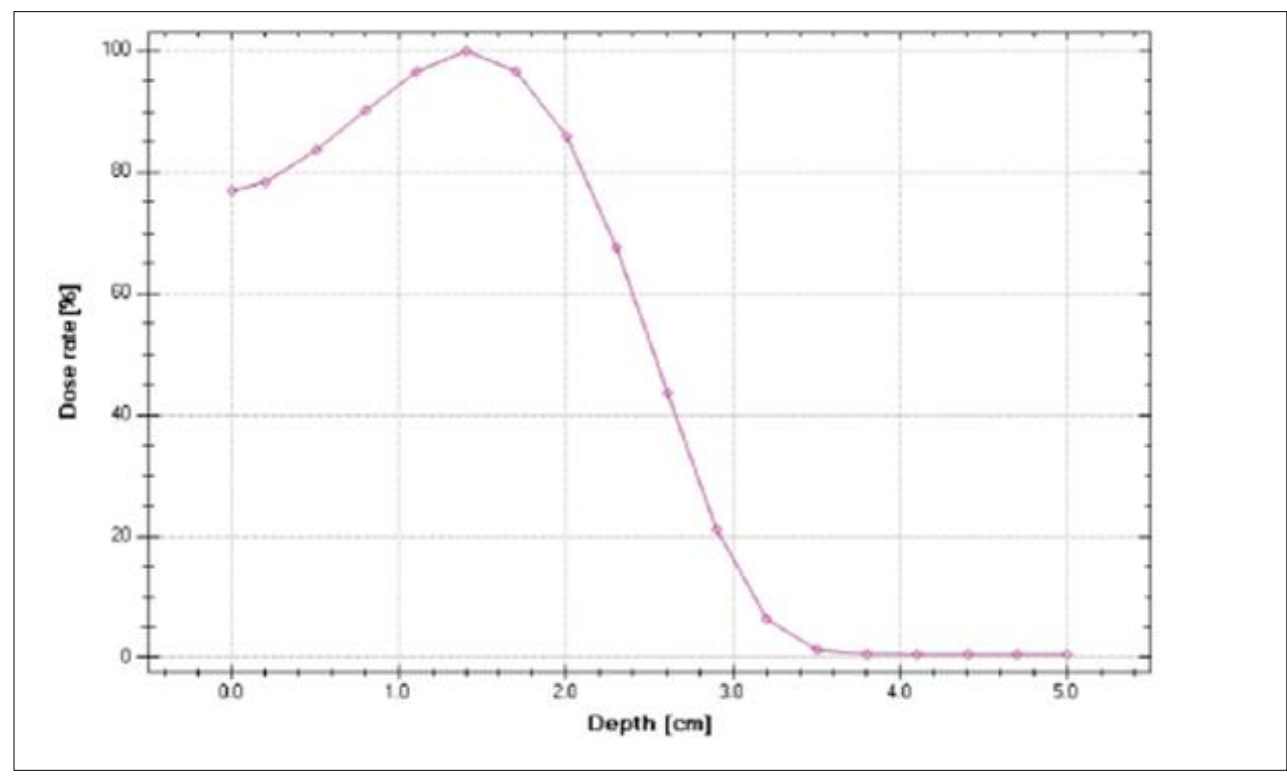

(a) PDD profile scanned at $6 \mathrm{MeV}$

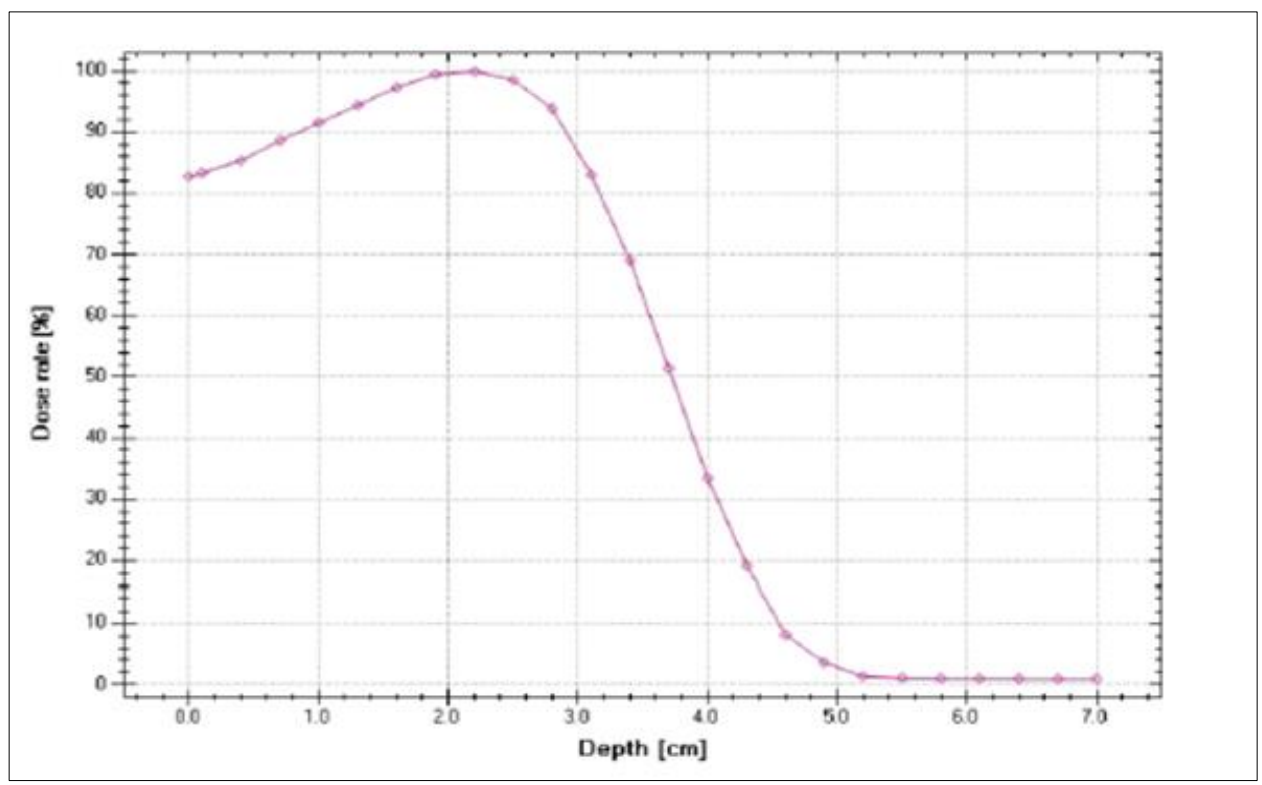

(b) PDD profile scanned at $9 \mathrm{MeV}$ 


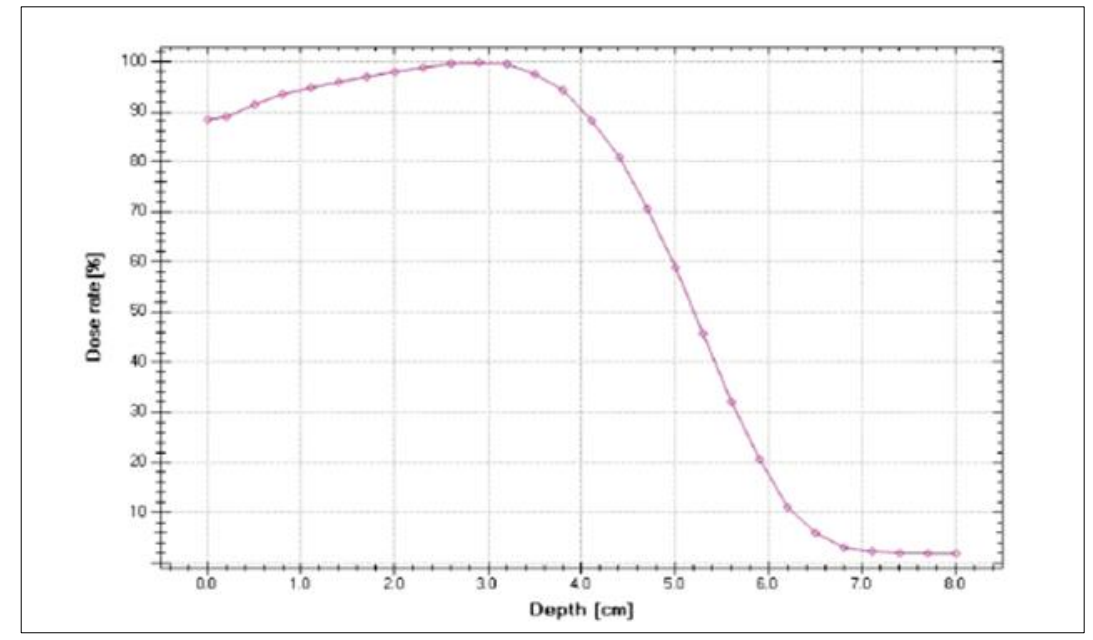

(c) PDD profile scanned at $12 \mathrm{MeV}$

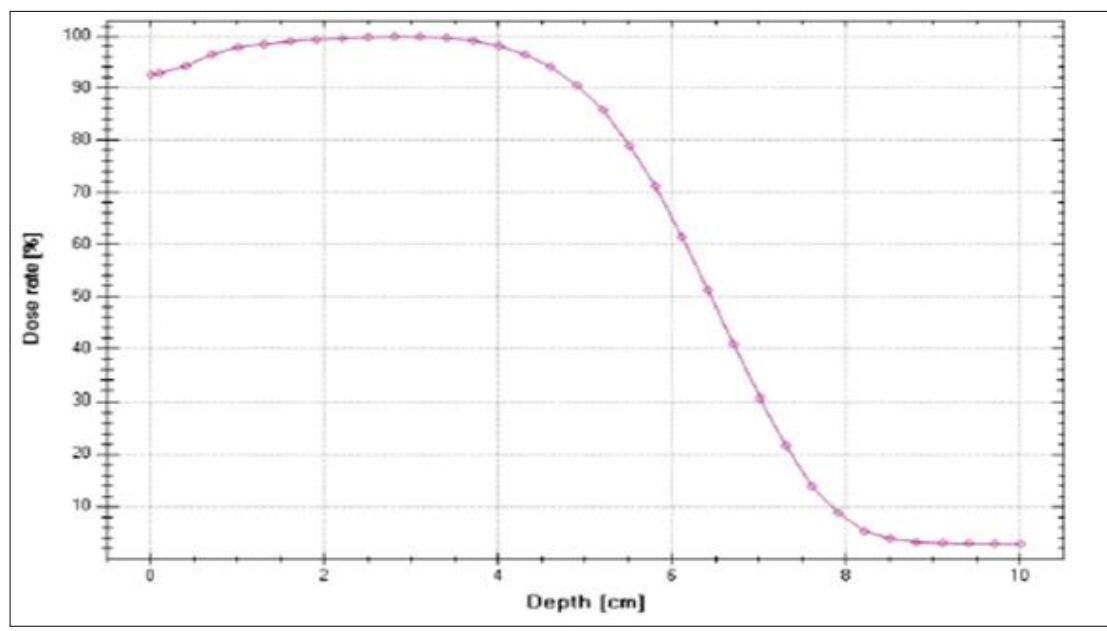

(d) PDD profile scanned at $15 \mathrm{MeV}$

Figure 4 The PDD curves of Electron beams scanned at: (a) $6 \mathrm{MeV}$, (b) $9 \mathrm{MeV}$, (c) $12 \mathrm{MeV}$, (d) $15 \mathrm{MeV}$

The corresponding measured data of the PDDs for the Electron beams of 6, 9,12 and $15 \mathrm{MeV}$ are presented in Table 18. In the measured PDDs, consistency was observed with manufacturer's estimations on the measured $90 \%, 80 \%$, and $50 \%$ PDDs at various depths of ionization of the 6, 9, 12 and $15 \mathrm{MeV}$ Electron energies.

Table 18 The PDDs at various Electron energies

\begin{tabular}{|c|c|c|c|}
\hline \multirow{2}{*}{ Beam energy (MeV) } & \multicolumn{3}{|c|}{ Percent of depth dose } \\
\cline { 2 - 4 } & $90 \%$ & $80 \%$ & $50 \%$ \\
\cline { 2 - 4 } & \multicolumn{3}{|c|}{ Depth of ionization (cm) } \\
\hline 6 & 1.87 & 2.09 & 2.52 \\
\hline 9 & 2.91 & 3.17 & 3.72 \\
\hline 12 & 4.02 & 4.43 & 5.20 \\
\hline 15 & 4.95 & 5.47 & 6.44 \\
\hline
\end{tabular}


3.2.3. Profile Flatness and Symmetry of Electron beams

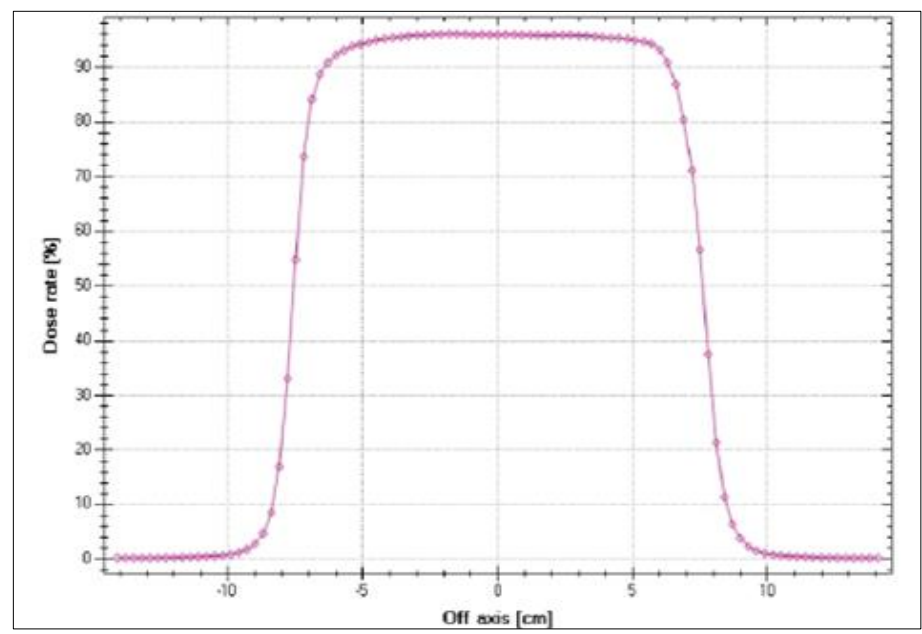

(a) Flatness and Symmetry Profile scanned at $6 \mathrm{MeV}$

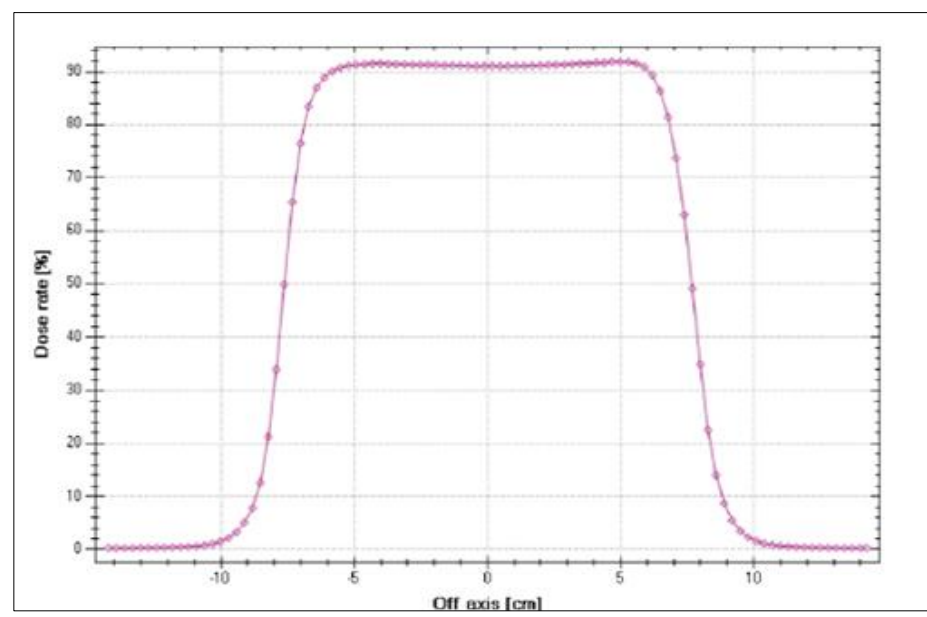

(b) Flatness and Symmetry Profile scanned at $9 \mathrm{MeV}$

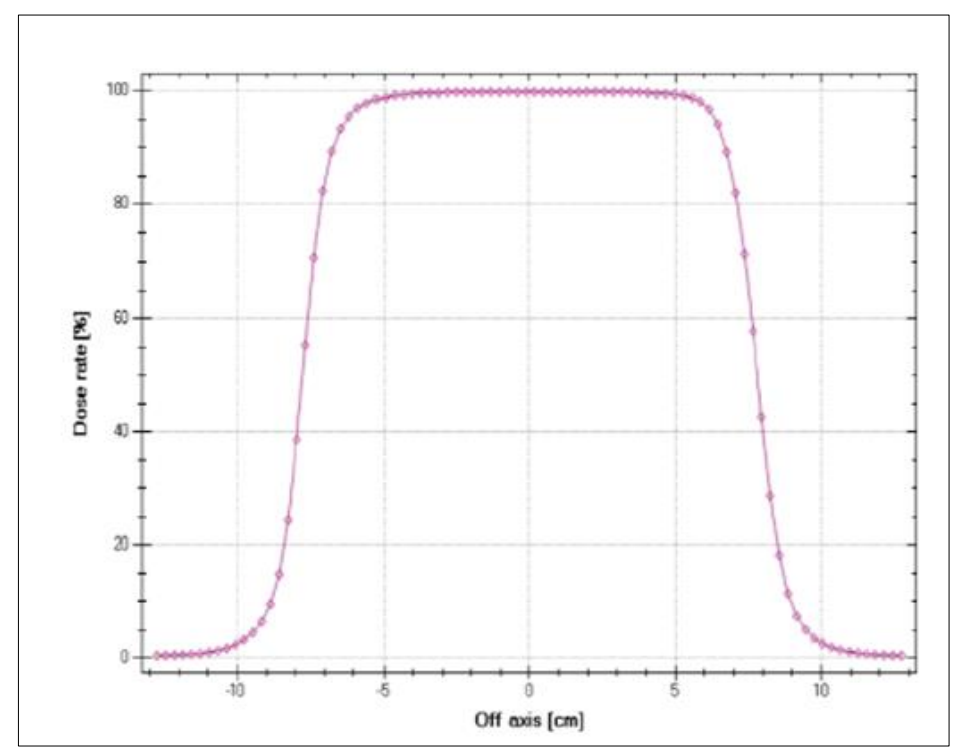

(c) Flatness and Symmetry Profile scanned at $12 \mathrm{MeV}$ 


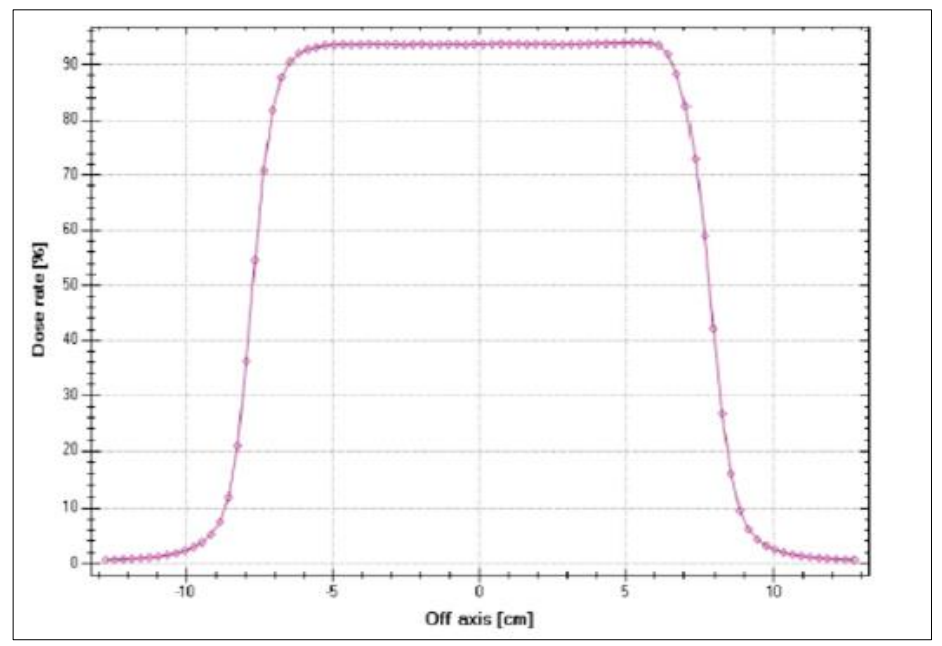

(d) Flatness and Symmetry Profile scanned at $15 \mathrm{MeV}$

Figure 5 The Flatness and Symmetry curves of the Electron beams scanned at (a) $6 \mathrm{MeV}$, (b) $9 \mathrm{MeV}$, (c) $12 \mathrm{MeV}$, (d) 15 $\mathrm{MeV}$ energies

The Flatness and Symmetry of Electron beams at various energies were verified at $100 \mathrm{~cm}$ SSD and 200MU using an IBA Compact Chamber (0.13cc IBA CC13). The Flatness and Symmetry curves of the Electron beams scanned at 6, 9, 12, 15 $\mathrm{MeV}$ energies are shown in Fig. 5 (a, b, c, d). The Profile flatness for $6 \mathrm{MeV}, 9 \mathrm{MeV}, 12 \mathrm{MeV}$ and $15 \mathrm{MeV}$ Electron energies were found to be $2.2 \%, 1.8 \%, 2.4 \%$ and $1.40 \%$ respectively. The Profile Symmetry for the $6 \mathrm{MeV}, 9 \mathrm{MeV}, 12 \mathrm{MeV}$ and $15 \mathrm{MeV}$ energies were found to be $1.2 \%, 1.1 \%, 1.2 \%$ and $1.40 \%$ respectively. The AAPM TG-40 recommended tolerances of the Profile Flatness and Symmetry are up to $\pm 3 \%$ and $\pm 3 \%$ for $6 \mathrm{MeV}, 9 \mathrm{MeV}, 12 \mathrm{MeV}$ and $15 \mathrm{MeV}$ Electron energy. Therefore, the investigated Profile flatness and symmetry were found within the tolerant range of the AAPM TG-40 scale.

The corresponding verified parameters of flatness and symmetry evaluations for the Electron beams of $6 \mathrm{MeV}, 9 \mathrm{MeV}$, $12 \mathrm{MeV}$ and $15 \mathrm{MeV}$ energies are presented in Table 19.

Table 19 The Flatness and Symmetry evaluation at various Electron energies

\begin{tabular}{|c|c|c|c|l|}
\hline Beam Energy (MeV) & Cone / Applicator $\left.\mathbf{( c m}^{2}\right)$ & Symmetry & Flatness & Penumbra \\
\hline 6 & $10 \times 10$ & $1.20 \%$ & $2.20 \%$ & $0.95 \mathrm{~cm}-1.15 \mathrm{~cm}$ \\
\hline 9 & $10 \times 10$ & $1.10 \%$ & $1.80 \%$ & $1.21 \mathrm{~cm}-1.31 \mathrm{~cm}$ \\
\hline 12 & $10 \times 10$ & $1.20 \%$ & $2.40 \%$ & $1.27 \mathrm{~cm}-1.40 \mathrm{~cm}$ \\
\hline 15 & $10 \times 10$ & $1.40 \%$ & $1.20 \%$ & $1.08 \mathrm{~cm}-1.18 \mathrm{~cm}$ \\
\hline
\end{tabular}

\subsubsection{Electron Cone Ratios}

Electron Cone ratios at a given Electron energy were determined by comparing the relative ionization at the depth of maximum dose for a given cone relative to that for the $15 \times 15 \mathrm{~cm}^{2}$ cone. All Measurement were performed at $100 \mathrm{~cm}$ SSD and 200MU using an IBA Compact Chamber (0.13cc IBA CC13). The measured Electron Cone ratios for various energies and Applicators are presented in Table 20. 
World Journal of Advanced Engineering Technology and Sciences, 2021, 03(01), 041-059

Table 20 The Electron Cone ratios for various energies and Cone/Applicators

\begin{tabular}{|c|c|c|c|c|c|c|}
\hline $\begin{array}{c}\text { Beam } \\
\text { energy } \\
(\mathrm{MeV})\end{array}$ & $\begin{array}{c}\text { Cone / } \\
\text { Applicator } \\
\left(\mathrm{cm}^{2}\right)\end{array}$ & Depth & $\mathrm{M}_{1 \times 10^{-8}} \mathrm{C}$ & $\mathrm{M}_{2 \times 10^{-8}} \mathrm{C}$ & Average & $\begin{array}{l}\text { Cone } \\
\text { Factor }\end{array}$ \\
\hline \multirow{5}{*}{6} & $6 \times 6$ & 1.33 & 0.772 & 0.77 & 0.771 & 0.965 \\
\hline & $10 \times 10$ & 1.33 & 0.797 & 0.797 & 0.797 & 0.997 \\
\hline & $15 \times 15$ & 1.33 & 0.799 & 0.798 & 0.799 & 1.000 \\
\hline & $20 \times 20$ & 1.33 & 0.808 & 0.807 & 0.808 & 1.011 \\
\hline & $25 \times 25$ & 1.33 & 0.805 & 0.805 & 0.805 & 1.008 \\
\hline \multirow{5}{*}{9} & $6 \times 6$ & 2.09 & 0.804 & 0.804 & 0.804 & 0.985 \\
\hline & $10 \times 10$ & 2.09 & 0.817 & 0.817 & 0.817 & 1.001 \\
\hline & $15 \times 15$ & 2.09 & 0.816 & 0.816 & 0.816 & 1.000 \\
\hline & $20 \times 20$ & 2.09 & 0.806 & 0.806 & 0.806 & 0.988 \\
\hline & $25 \times 25$ & 2.09 & 0.791 & 0.791 & 0.791 & 0.969 \\
\hline \multirow{5}{*}{12} & $6 \times 6$ & 2.89 & 0.819 & 0.82 & 0.82 & 0.986 \\
\hline & $10 \times 10$ & 2.89 & 0.838 & 0.836 & 0.837 & 1.007 \\
\hline & $15 \times 15$ & 2.89 & 0.832 & 0.831 & 0.832 & 1.000 \\
\hline & $20 \times 20$ & 2.89 & 0.82 & 0.818 & 0.819 & 0.985 \\
\hline & $25 \times 25$ & 2.89 & 0.798 & 0.796 & 0.797 & 0.959 \\
\hline \multirow{5}{*}{15} & $6 \times 6$ & 3.52 & 0.827 & 0.828 & 0.828 & 0.991 \\
\hline & $10 \times 10$ & 3.52 & 0.845 & 0.844 & 0.845 & 1.011 \\
\hline & $15 \times 15$ & 3.52 & 0.835 & 0.835 & 0.835 & 1.000 \\
\hline & $20 \times 20$ & 3.52 & 0.822 & 0.822 & 0.822 & 0.984 \\
\hline & $25 \times 25$ & 3.52 & 0.798 & 0.798 & 0.798 & 0.956 \\
\hline
\end{tabular}

\section{Conclusion}

The dosimetric characteristics is verified for the megavoltage Photon and Electron beams of a medical LINAC to be useful for treatment planning in a wide range of conditions. Some key parameters, namely PDDs, Profiles, beam Output, and Output Factors are determined with a series of Field Sizes (FSs). The measured parameters of the QA process are authenticated in reference to the BJR-25 [12] and The Task Group-51 (AAPM TG-51) [15]. Therefore, accurately measured PDDs, Profiles, beam Output, and Output Factors of Photon and Electron beams indicate its efficacy for the treatment purpose of a LINAC system.

The Photon beam outputs ( $\left.D_{\max } / \mathrm{MU}\right)$ are standardized to the tuned doses of 1.0002 and $0.9988 \mathrm{cGy} / \mathrm{MU}$ for $6 \mathrm{MV}$ and $10 \mathrm{MV}$ Photon beams, respectively; which significantly satisfies the beam output standardization criteria of $1 \mathrm{cGy} / \mathrm{MU}$ standard output dose.

The measured PDDs with different field sizes for both 6MV and 10MV Photon beams are authenticated in reference to the BJR-25 that indicates reasonably a good agreement. In addition, these measured PDDs and Profiles with different field sizes for both 6MV and 10MV Photon beams are verified in terms of the TPS calculated PDDs and Profile, and the variation is found to be less than $\pm 2 \mathrm{~mm}$. 
The Profile Flatness and Symmetry are investigated with FS $(10 \times 10) \mathrm{cm}^{2}$ for $6 \mathrm{MV}$ and $10 \mathrm{MV}$ Photon beams. The Flatness and Symmetry are found to be within the AAPM TG- 40 tolerance ranges of up to $\pm 3 \%$ and $\pm 2 \%$ for $6 \mathrm{MV}$ and 10MV Photon beams, respectively [9]. Therefore, Flatness and Symmetry of Photon beams satisfies the required accuracy of clinical applications.

The Electron beam outputs $\left(D_{w, \max } / \mathrm{MU}\right)$ are standardized to the tuned doses of $1.0031,1.0049,1.0014,0.9985 \mathrm{cGy} / \mathrm{MU}$ for $6 \mathrm{MeV}, 9 \mathrm{MeV}, 12 \mathrm{MeV}$ and $15 \mathrm{MeV}$, respectively; which reasonably satisfies the standardization criteria of $1 \mathrm{cGy} / \mathrm{MU}$ standard output dose.

The Profile Flatness and Symmetry are found to be within the AAPM TG- 40 tolerance ranges of up to $\pm 3 \%$ and $\pm 3 \%$ for $6 \mathrm{MeV}, 9 \mathrm{MeV}, 12 \mathrm{MeV}$ and $15 \mathrm{MeV}$ Electron beams, respectively; hence reasonable accuracy is observed to be useful in clinical applications with LINAC Electron beams.

The Electron Cone ratios at various Electron energies are determined. The Cone Factors are found to be in the range of $0.965-1.011,0.985-1.001,0.985-1.007$, and $0.984-1.011$ at energies $6 \mathrm{MeV}, 9 \mathrm{MeV}, 12 \mathrm{MeV}$ and $15 \mathrm{MeV}$ respectively with FS ranging from $6 \times 6 \mathrm{~cm}^{2}$ to $25 \times 25 \mathrm{~cm}^{2}$.

\section{Compliance with ethical standards}

\section{Acknowledgments}

This research work did not receive grant from any organization. Nevertheless, the technical support was afforded by Delta Hospital Limited throughout the execution of this research work.

\section{Disclosure of conflict of interest}

There is no conflict of interest declared on this research article.

\section{References}

[1] Akulapalli Sudhakar. History of Cancer, Ancient and Modern Treatment Methods. J Cancer Sci Ther. 2009; 1(2): $1-4$.

[2] Baskar R, Lee KA, Yeo R, Yeoh K-W. Cancer and Radiation Therapy: Current Advances and Future Directions. Int J Med Sci. 2012; 9(3): 193-9.

[3] Das IJ, Ding GX, Ahnesjö A. Small fields: Nonequilibrium radiation dosimetry. Med Phys. 2008; 35: 206-15.

[4] Scott AJ, Nahum AE, Fenwick JD. Using a Monte Carlo model to predict dosimetric properties of small radiotherapy photon fields. Med Phys. 2008; 35: 4671-84.

[5] Henry FG, Ravikumar M, Sathiyan S, Ganesh KM, Retna YP, Varatharaj C. Analysis of small field percent depth dose and profiles: Comparison of measurements with various detectors and effects of detector orientation with different jaw settings. Journal of Medical Physics. 2016; 41(1).

[6] Tiru S Subramanian, JP Gibbons, J William RH. Moderator Linear accelerators used for IMRT should be designed as small field, high intensity, intermediate energy unit. Med. Phys. 2002; 29(11).

[7] Jing Hao, Anthony Magnelli, Andrew Godley, Jennifer S Yu. Use of a Linear Accelerator for Conducting In Vitro Radiobiology experiments. J Vis Exp. Author manuscript. 2020.

[8] Bethesda MD. International Commission on radiation Units and Measurements (ICRU), Determination of Absorbed Dose in a Patient Irradiated by Beams of X or Gamma rays in Radiotherapy Procedures. 1976; 24.

[9] Gerald J Kutcher, Lawrence Coia, Michael Gillin, William F Hanson, et al. Comprehensive QA for radiation oncology: Report of AAMP radiation therapy committee. Task Group-40 (TG-40) protocol of American Association of Physicists in Medicine (AAPM). Med. Phys. 1994; 21(4).

[10] Eric E Klein, Joseph Hanley, John Bayouth, fabg-Fang Yin, et. al. Quality assurance of medical accelerators. Task Group-142 (TG-142) protocol of American Association of Physicists in Medicine (AAPM). Med. Phys. 2009; 36(9).

[11] Sahoo SK, Rath AK, Mukharjee RN, Mallick B. Commissioning of a Modern LINAC for Clinical Treatment and Material Research. International Journal of Trends in Interdisciplinary Studies. 2012; 10. 
[12] BJR Supplement 25 "British Journal of Radiotherapy (BJR-25)". Central Axis Depth Dose Data for Use in Radiotherapy. British Journal of Radiology. 1996; Suppl. 25.

[13] Podgorsak EB. Radiation Physics for Medical Physicists. 2010; 110-112.

[14] IEC (International Electrical Commission). Medical electrical equipment-Dosimeters with ionization chambers as used in radiotherapy. 2016; 3.1.

[15] Peter R Almond, Peter J Biggs, BM Coursey, WF Hanson, et. al. Clinical reference dosimetry of energy photon and electron beams. Task Group-51 (TG-51) protocol of American Association of Physicists in Medicine (AAPM). Med. Phys. 1999; 26(9).

[16] IEC (International Electrical Commission). Medical electrical equipment-Medical electron acceleratorsFunctional performance characteristics. 2007; 2.0.

[17] Bruce J Gerbi, Jhon A Antolak, F Christopher Deibel, David S Followill, et al. Recommendations for clinical electron beam dosimetry: Supplement to the recommendations of Task Group-25. Task Group-25 (TG-25) protocol of American Association of Physicists in Medicine (AAPM). Med. Phys. 2009; 36(7). 